\title{
Perioperative lung ultrasound pattern changes in patients undergoing gynecological procedures - a prospective observational study
}

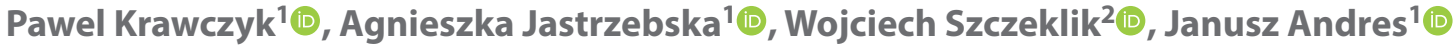 \\ ${ }^{1}$ Department of Anesthesiology and Intensive Care Medicine, Jagiellonian University Medical College, Cracow, Poland \\ ${ }^{2}$ Department of Intensive Care and Perioperative Medicine, Jagiellonian University Medical College, Cracow, Poland
}

\begin{abstract}
Objectives: General anesthesia and positive pressure ventilation are associated with perioperative pulmonary complications. Lung ultrasound (LUS) is a method used to evaluate lung parenchyma. The purpose of this study was to evaluate LUS patterns in a cohort of women undergoing gynecological surgery with uncomplicated general anesthesia.

Material and methods: Patients were assessed according to the 8-zone LUS assessment protocol used to detect lung sliding, A-lines, B-lines, interstitial syndrome and lung consolidation. Each patient was screened at specific time intervals: before induction of anesthesia, at induction, 30 and 60 minutes after induction and within two hours after recovery.

Results: A total of 99 patients undergoing gynecological surgery with uneventful anesthesia from November 2017 to November 2018 were included in this study. A total of 426 LUS records were retained for further analysis. Overall, no significant changes to patients' $\mathrm{A}$-line appearance were detected, regardless of the time of assessment. There was, however, an increase in the number of $B$-lines at the screening times of 30 and 60 minutes after induction, as compared to initial assessments $(p=0.011$ and $p<0.001$ respectively), and an increase in the number of positive regions ( $\geq 3$ B-lines) at 30 and 60 minutes after induction and after recovery, as compared to initial assessment $(p<0.001 ; p<0.001$ and $p=0.001$ respectively).

Conclusions: An uneventful anesthesia may predispose to abnormal LUS findings and should be considered while interpreting of LUS results in cases with perioperative pulmonary complications.
\end{abstract}

Key words: lung ultrasound; perioperative care; B-lines; interstitial syndrome

Ginekologia Polska 2021; 92, 4: 271-277

\section{INTRODUCTION}

Lung ultrasound (LUS) is a well-recognized diagnostic tool. One benefit of this quick, easily repeated bedside assessment is to help diagnose possible reasons for patient deterioration, including alveolar consolidation, pulmonary edema, pneumothorax and pleural effusion [1-3]. There is a short learning curve when learning to use LUS technique, and high intra-and inter-observer reproducibility confirmed in rapid training for gynecologists and obstetricians [4].

There is growing evidence that LUS is a very useful tool for gynecologists and obstetricians. Published evidence concern COVID-19 obstetric patients, pre-eclamptic patients or pregnant patients during the last gestational weeks and labor where lung ultrasound is often performed by gynecologists and obstetricians [5-10]. Perioperative setting may also involve need of LUS assessment in case of respiratory complications.
The incidence of postoperative pulmonary complications (PPC) may be even up to $59 \%$ depending on the surgical patient population [11, 12]. Recently published reviews indicated a need for deeper exploration of perioperative ultrasound including scanning protocols, time intervals when scanning should occur and patient benefits [13].

\section{Objectives}

The goal of this study was to assess the LUS pattern and its changes in patients undergoing general anesthesia with positive pressure ventilation. The hypothesis behind the study was that even uneventful general anesthesia with positive pressure ventilation may cause visible changes in the LUS pattern.

\section{MATERIAL AND METHODS}

After receiving approval from the Ethics Committee of Jagiellonian University, Cracow, Poland (approval number: 
122.6120.142.2016; 23 June 2016), this prospective observational study was conducted in a single university hospital. All study participants gave written, informed consent to participate in the study before enrolment.

Patient participation was voluntary. The study population was composed of female patients scheduled for elective, gynecological procedures using general anesthesia and positive pressure ventilation. Patients chosen for this study were required to be 18 years of age or older and able to provide informed, written consent. Patients were excluded from this study if receiving pregnancy-related surgery, if the research personnel was unable to obtain all LUS assessments (i.e., the initial assessment before induction of anesthesia or any of the following within the study protocol time frames) or if patients were experiencing any respiratory or circulatory complications at the time of the study. Parameters of interest were assessed using standard perioperative monitoring devices (the Datex Ohmeda S/5 Aespire Patient Monitor, GE Healthcare Helsinki, Finland).

\section{Data collection}

Baseline patient characteristics were collected including age, weight, height, body mass index (BMI), ideal body weight (IBW) according to the Lorenz formula [14], American Society of Anesthesiologists Physical Status (ASA) [15], positive end expiratory pressure (PEEP), peak inspiratory pressure (PIP), perioperative fluids management, fraction of inspired oxygen $\left(\mathrm{FiO}_{2}\right)$ during induction and recovery from anesthesia, intra-abdominal carbon dioxide pressure during laparoscopy, type of surgery (laparoscopy, laparotomy, hysteroscopy), airway management (tracheal intubation versus supraglottic airway), patient's use of muscle relaxants, intravenous fluid volume, and duration of anesthesia. The LUS and parameters of interest were recorded before induction of anesthesia, at induction of anesthesia and 30 and 60 minutes after induction according to duration of surgery. The last LUS assessment was conducted in the recovery room within two hours after surgery.

\section{Anesthesia}

Since it was an observational study no changes were done to the anesthesia and intraoperative ventilator protocols and management used in the institution. Thirty minutes before induction of anesthesia, patients received $2 \mathrm{mg}$ of midazolam intravenously. General anesthesia was induced with propofol 2-3 $\mathrm{mg} \mathrm{kg}^{-1}$, fentanyl 1-2 $\mathrm{g} \mathrm{kg}^{-1}$ and, in cases of muscle relaxation, rocuronium $0.6 \mathrm{mg} \mathrm{kg}^{-1}$. Maintenance of anesthesia was performed with oxygen/air mixture $\left(\mathrm{FiO}_{2} \mathrm{0.4}\right)$ and sevoflurane (0.8-1.2 of age adjusted expiratory minimal alveolar concentration), fresh gas flow $31 \mathrm{~min}^{-1}$. Ventilation rate (volume-controlled ventilation, I:E ratio of
$1: 2)$ was adjusted to maintain normocapnia with a tidal volume of $6-8 \mathrm{ml} \mathrm{kg}^{-1}$ IBW and PEEP of 2-5 $\mathrm{cm} \mathrm{H}_{2} \mathrm{O}$. An infusion of intravenous crystalloid fluid therapy was initiated on each patient before induction of anesthesia. Patients received 0.5-1 mg of atropine and $2 \mathrm{mg}$ of neostigmine intravenously to reverse the neuromuscular block.

\section{Ultrasound protocol}

There are various LUS assessment protocols that allow qualitative and quantitative assessment of dynamically changing LUS artefacts [16-18]. However, not all protocols are relevant to surgery in the supine position. For this reason, the 8-zone protocol, as defined in the "International evidence-based recommendations for point of care lung ultrasound" [19], was chosen for this study. All patients' lungs were assessed for the presence of lung sliding, A-lines, B-lines, interstitial syndrome [two or more bilateral positive regions (presence of three or more B-lines)], lung consolidation and pleural effusion using the 8-zone protocol [19]. For the initial and last LUS assessment, the patients were in a semi-recumbent position. During anesthesia, the patients were positioned in the Trendelenburg position according to their specific surgical needs.

All ultrasound assessments were conducted independently by two certified physicians (PK and AJ) experienced in the administration of LUS assessments. All ultrasound assessments were performed using the SignosRT instrument (Signostic Limited, Clovely Park SA, Australia) with a sector probe with lung pre-set and $10 \mathrm{~cm}$ of depth $(3.0-5.0 \mathrm{MHz}$; Signostic Limited, Clovely Park SA, Australia).

\section{Statistical analysis}

A power analysis was conducted using data from preliminary study. A sample of 56 patients showed an overall $80 \%$ power for detecting the differences with a two tailed alpha of 0.05 , as represented by the presence of three or more B-lines, 30 minutes after the "before induction" time point. In cases of normally distributed data, continuous data are presented as a mean value with standard deviation. Non-normally distributed data are presented as a median value with interquartile range. Normality was assessed using the Shapiro-Wilk test. Discrete data are presented as frequency and percentage.

The changes in the number of fields for A-lines, B-lines and $\geq 3$ B-lines were assessed by pairing Wilcoxon tests with Bonferroni corrections over multiple comparisons. Comparisons of two repeated measures of dichotomic variables were made using the McNemar test. Analyses were conducted using R software (ver. 3.5.1; R Development Core Team, Austria, Vienna) [20]. Results with a $p$ value $<0.05$ were considered significant. 
Table 1. Basic patient characteristics $(n=99)$

\begin{tabular}{|c|c|c|}
\hline Variables & \multicolumn{2}{|l|}{ Value } \\
\hline Age [years] median (IQR) & \multicolumn{2}{|l|}{$39(16)$} \\
\hline Weight [kg] median (IQR) & \multicolumn{2}{|l|}{$63(19)$} \\
\hline Height $[\mathrm{cm}]$ mean \pm SD & \multicolumn{2}{|l|}{$165.21 \pm 6.51$} \\
\hline BMI [kg/m²] median (IQR) & \multicolumn{2}{|l|}{$24.01(7.04)$} \\
\hline $\mathrm{IBW}[\mathrm{kg}]$ mean \pm SD & \multicolumn{2}{|l|}{$57.61 \pm 3.25$} \\
\hline Fluids administrated i.v. [mL] median (IQR) & \multicolumn{2}{|l|}{$850(250)$} \\
\hline Intraabdominal laparoscopic $\mathrm{CO}_{2}$ pressure $\left[\mathrm{cm} \mathrm{H}_{2} \mathrm{O}\right]$ median (IQR) & \multicolumn{2}{|l|}{$14(1)$} \\
\hline PIP $\left[\mathrm{cm} \mathrm{H}_{2} \mathrm{O}\right]$ median (IQR) & \multicolumn{2}{|l|}{$17(8)$} \\
\hline TV [mL] median (IQR) & \multicolumn{2}{|l|}{$425(50)$} \\
\hline $\mathrm{TV} / \mathrm{IBW}[\mathrm{mL} / \mathrm{kg}]$ mean $\pm \mathrm{SD}$ & \multicolumn{2}{|l|}{$7.35 \pm 0.74$} \\
\hline \multirow{2}{*}{ ASA (n) } & ASA 1-2 & $93(93 \%)$ \\
\hline & ASA 3 & $6(6 \%)$ \\
\hline \multirow{4}{*}{ Type of surgery (n) } & Laparoscopy & $71(71 \%)$ \\
\hline & Laparotomy & $14(14 \%)$ \\
\hline & Hysteroscopy & $13(13 \%)$ \\
\hline & Missing data & $1(1 \%)$ \\
\hline \multirow{2}{*}{ Muscle relaxants use } & No & $13(13 \%)$ \\
\hline & Yes & $86(86 \%)$ \\
\hline \multirow{2}{*}{ Duration of surgery } & $\leq 1 \mathrm{~h}$ & $55(55 \%)$ \\
\hline & $>1 \mathrm{~h}$ & $44(44 \%)$ \\
\hline \multirow{2}{*}{$\mathrm{FiO}_{2}$ during induction and recovery from anesthesia } & 0.8 & $20(20 \%)$ \\
\hline & 1.0 & $76(76 \%)$ \\
\hline \multirow{2}{*}{$\operatorname{PEEP}\left(\mathrm{cmH}_{2} \mathrm{O}\right)$} & PEEP $>3$ & $50(50 \%)$ \\
\hline & PEEP $\leq 3$ & $49(49 \%)$ \\
\hline
\end{tabular}

$\mathrm{BMI}$ - body mass index; IBW — ideal body weight; $\mathrm{CO}_{2}$ — carbon dioxide; PIP — peak inspiratory pressure; TV — tidal volume; ASA — American Society of Anesthesiologists Physical Status; $\mathrm{FiO}_{2}$ — fraction of inspired oxygen; PEEP — positive end expiratory pressure; IQR — interquartile range; SD — standard deviation

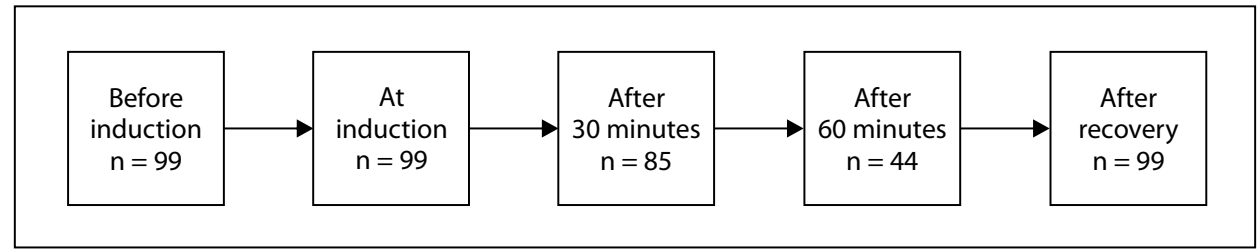

Figure 1. The study flowchart

\section{RESULTS}

One hundred patients were included in the study on a voluntary basis, from November 2017 to November 2018. One patient was excluded from the initial study group due to major bleeding and cardiovascular instability during surgery.

The following surgical procedures were performed: laparoscopic removal of non-malignant ovarian tumor - 35, laparoscopic supracervical hysterectomy - 23, diagnostic hysteroscopy -13 , laparoscopic diagnosis of infertility -10 , myomectomy - 8, gynecological oncology -6 , removal of tumor in a cesarean scar -1 , laparoscopic diagnosis of pelvic pain syndrome -1 , oncofertility -1 , sacrofixation in pelvic organ prolapse -1 .

Table 1 presents the patient characteristics. Figure 1 (study flowchart) presents the number of LUS assessments performed at specified time intervals. Four hundred and twenty-six records were collected for further analysis. All patients' vital signs were stable within normal ranges during surgery, and none demonstrated short-term PPC. Lung 
Table 2. Frequency (percentage) of A-line, B-line, positive regions and consolidations detection according to the probe location during the 8-zone lung ultrasound assessment [19]

\begin{tabular}{|c|c|c|c|c|c|c|c|c|}
\hline Variables & RUA & RLA & RBL & RUL & LUA & LLA & LBL & LUL \\
\hline \multicolumn{9}{|l|}{ A-lines } \\
\hline Before induction & 80 (80.81\%) & 79 (79.8\%) & $65(65.66 \%)$ & $82(82.83 \%)$ & $68(68.69 \%)$ & 49 (49.49\%) & $73(73.74 \%)$ & $68(68.69 \%)$ \\
\hline After induction & $72(72.73 \%)$ & $62(62.63 \%)$ & 72 (72.73\%) & 75 (75.76\%) & $80(80.81 \%)$ & $54(54.55 \%)$ & 68 (68.69\%) & $69(69.7 \%)$ \\
\hline 30 minutes & $46(54.12 \%)$ & $54(63.53 \%)$ & $55(64.71 \%)$ & $57(67.06 \%)$ & $52(61.18 \%)$ & $44(51.76 \%)$ & $60(70.59 \%)$ & $55(64.71 \%)$ \\
\hline 60 minutes & $30(68.18 \%)$ & $25(56.82 \%)$ & $25(56.82 \%)$ & $25(56.82 \%)$ & $23(52.27 \%)$ & $24(54.55 \%)$ & $29(65.91 \%)$ & $29(65.91 \%)$ \\
\hline 2 hours after recovery & $60(60.61 \%)$ & $56(56.57 \%)$ & $66(66.67 \%)$ & $63(63.64 \%)$ & $55(55.56 \%)$ & $47(47.47 \%)$ & $59(59.6 \%)$ & $67(67.68 \%)$ \\
\hline \multicolumn{9}{|l|}{ B-lines } \\
\hline Before induction & $21(21.21 \%)$ & $25(25.25 \%)$ & $26(26.26 \%)$ & $23(23.23 \%)$ & $22(22.22 \%)$ & $16(16.16 \%)$ & $32(32.32 \%)$ & $23(23.23 \%)$ \\
\hline After induction & $24(24.24 \%)$ & $26(26.26 \%)$ & $25(25.25 \%)$ & $45(45.45 \%)$ & $29(29.29 \%)$ & $29(29.29 \%)$ & $37(37.37 \%)$ & $30(30.3 \%)$ \\
\hline 30 minutes & $37(43.53 \%)$ & $23(27.06 \%)$ & $20(23.53 \%)$ & $41(48.24 \%)$ & $31(36.47 \%)$ & $25(29.41 \%)$ & $39(45.88 \%)$ & $39(45.88 \%)$ \\
\hline 60 minutes & $25(56.82 \%)$ & $13(29.55 \%)$ & $13(29.55 \%)$ & $24(54.55 \%)$ & $16(36.36 \%)$ & $13(29.55 \%)$ & $31(70.45 \%)$ & $21(47.73 \%)$ \\
\hline 2 hours after recovery & $27(27.27 \%)$ & $33(33.33 \%)$ & $40(40.4 \%)$ & $29(29.29 \%)$ & $21(21.21 \%)$ & $24(24.24 \%)$ & $45(45.45 \%)$ & $41(41.41 \%)$ \\
\hline \multicolumn{9}{|c|}{ Positive regions ( $\geq 3$ B-lines) } \\
\hline Before induction & $4(4.04 \%)$ & $4(4.04 \%)$ & $2(2.02 \%)$ & $6(6.06 \%)$ & $3(3.03 \%)$ & $1(1.01 \%)$ & $0(0 \%)$ & $1(1.01 \%)$ \\
\hline After induction & $3(3.03 \%)$ & $2(2.02 \%)$ & $2(2.02 \%)$ & $9(9.09 \%)$ & $5(5.05 \%)$ & $0(0 \%)$ & $4(4.04 \%)$ & $7(7.07 \%)$ \\
\hline 30 minutes & $12(14.12 \%)$ & $5(5.88 \%)$ & $2(2.35 \%)$ & $14(16.47 \%)$ & $12(14.12 \%)$ & $0(0 \%)$ & $10(11.76 \%)$ & $16(18.82 \%)$ \\
\hline 60 minutes & $15(34.09 \%)$ & $5(11.36 \%)$ & $2(4.55 \%)$ & $13(29.55 \%)$ & $5(11.36 \%)$ & $0(0 \%)$ & $3(6.82 \%)$ & $8(18.18 \%)$ \\
\hline 2 hours after recovery & $10(10.1 \%)$ & $12(12.12 \%)$ & $12(12.12 \%)$ & $12(12.12 \%)$ & $5(5.05 \%)$ & $7(7.07 \%)$ & $0(0 \%)$ & $10(10.1 \%)$ \\
\hline \multicolumn{9}{|l|}{ Consolidation } \\
\hline Before induction & $0(0 \%)$ & $0(0 \%)$ & $0(0 \%)$ & $0(0 \%)$ & $0(0 \%)$ & $1(1.01 \%)$ & $21(21.21 \%)$ & $0(0 \%)$ \\
\hline After induction & $0(0 \%)$ & $0(0 \%)$ & $1(1.01 \%)$ & $0(0 \%)$ & $0(0 \%)$ & $17(17.17 \%)$ & $0(0 \%)$ & $0(0 \%)$ \\
\hline 30 minutes & $0(0 \%)$ & $0(0 \%)$ & $0(0 \%)$ & $0(0 \%)$ & $0(0 \%)$ & $12(14.12 \%)$ & $0(0 \%)$ & $1(1.18 \%)$ \\
\hline 60 minutes & $0(0 \%)$ & $0(0 \%)$ & $0(0 \%)$ & $0(0 \%)$ & $0(0 \%)$ & $6(13.64 \%)$ & $0(0 \%)$ & $1(2.27 \%)$ \\
\hline 2 hours after recovery & $0(0 \%)$ & $0(0 \%)$ & $0(0 \%)$ & $0(0 \%)$ & $1(1.01 \%)$ & $1(1.01 \%)$ & $23(23.23 \%)$ & $2(2.02 \%)$ \\
\hline
\end{tabular}

Probe locations during the LUS assessment: RUA — right upper anterior; RLA — right lower anterior; RUL — right upper lateral; RBL — right basal lateral; LUA — left upper anterior; LLA — left lower anterior; LUL — left upper lateral; LBL — left basal lateral. Data are presented as frequencies (percentages)

sliding was present on all ultrasound areas of assessment. Records showed neither pleural effusion nor subcutaneous emphysema. Table 2 and Table 3 show the frequency (percentage) of ultrasound artefact detected during anesthesia and any changes to artefact over time.

Overall, no significant changes were detected in patients' A-line appearance, regardless of time of assessment. There was a general increase in the number of B-lines present at 30 and 60 minutes after induction, as compared to the initial assessment $(p=0.011$ and $p<0.001$ respectively), and an increase in the number of positive regions ( $\geq 3$ B-lines) at 30 and 60 minutes after induction and after recovery, as compared to the initial assessment $(p<0.001$ and $p<0.001$ and $p=0.001$ respectively). There was also an increase in the number of positive regions ( $\geq 3 \mathrm{~B}$-lines) $30 \mathrm{~min}$ utes after induction, as compared to the number of positive regions recorded at induction $(p=0.001)$. The presence of at least one positive region was common and present in 26 (26\%) patients at induction, $56(66 \%)$ patients 30 minutes after induction, 37 (84\%) patients 60 minutes after induction and 44 (44\%) patients after recovery. At the time of 30 and 60 minutes after induction and after recovery there were more positive regions in upper than lower lung areas.

Lung consolidation was detected in single LUS assessment area in 21 pre-medicated patients before induction of anesthesia. After recovery twenty-three patients were found with consolidations in LUS. Their frequency of appearance did not reach statistical significance in the specific time frames.

Interstitial syndrome criteria [19] were met in six patients at different time frames: one before induction of anesthesia, one at induction, one at 30 minutes after induction. Three patients had interstitial syndrome after 60 minutes and within two hours after recovery. All six patients were offered $\mathrm{FiO}_{2} 1.0$ during induction and recovery from anesthesia. Basic characteristics of these patients are present in Table 4. Due to small sample size logistic regression was not performed in those cases. 
Table 3. Mean number of fields in 8-zone protocol with present artefacts in different time points

\begin{tabular}{|l|l|l|l|}
\hline Mean number of fields in 8-zone protocol & A-lines & B-lines & $\geq 3$ B-lines \\
\hline Before induction & 5.7 & 1.9 & 0.21 \\
\hline After induction & 5.58 & 2.47 & 0.32 \\
\hline 30 minutes & 4.98 & 3 & 0.84 \\
\hline 60 minutes & 4.77 & 3.55 & 1.16 \\
\hline $2 h$ after recovery & 4.78 & 2.63 & 0.69 \\
\hline Comparison & & & \\
\hline Before vs after induction $(n=99)$ & $p=1$ & $p=1$ & $p=0.886$ \\
\hline Before vs after 30 min $(n=85)$ & $p=0.58$ & $p=0.011$ & $p<0.001$ \\
\hline Before vs after 60 min $(n=44)$ & $p=0.321$ & $p<0.001$ & $p<0.001$ \\
\hline Before induction vs $2 h$ after recovery $(n=99)$ & $p=0.241$ & $p=0.583$ & $p=0.001$ \\
\hline After induction vs after 30 min $(n=85)$ & $p=1$ & $p=1$ & $p=0.001$ \\
\hline After 30 min vs after 60 min $(n=44)$ & $p=1$ & $p=1$ & $p=1$ \\
\hline After induction vs $2 h$ after recovery $(n=99)$ & $p=0.609$ & $p=1$ & $p=0.414$ \\
\hline
\end{tabular}

Table 4. Basic characteristics of the patient with interstitial syndrome detected in perioperative lung ultrasound

\begin{tabular}{|l|l|l|l|l|l|}
\hline Patient & Age (years) & ASA & Interstitial syndrome presence & Duration of anesthesia (minutes) & BMI $\left(\mathbf{k g} / \mathbf{m}^{2}\right)$ \\
\hline 1 & 35 & 1 & $\begin{array}{l}\text { After } 30 \text { minutes } \\
\text { After recovery }\end{array}$ & 85 & 18.3 \\
\hline 2 & 38 & 1 & $\begin{array}{l}\text { After recovery } \\
\text { Before anesthesia } \\
\text { At induction } \\
\text { After recovery }\end{array}$ & 45 & 30.0 \\
\hline 4 & 71 & 2 & After 60 minutes & 80 & 25.4 \\
\hline 5 & 33 & 1 & After 60 minutes & 150 & 19.5 \\
\hline 6 & 41 & 2 & After 60 minutes & 180 & 23.0 \\
\hline
\end{tabular}

ASA — American Society of Anesthesiologists Physical Status; BMI — body mass index

\section{DISCUSSION}

In the current study, we were able to identify frequent LUS pattern changes in a cohort of 99 women undergoing uneventful general anesthesia with positive pressure ventilation. There was a general increase in B-lines and positive region numbers ( $\geq 3 \mathrm{~B}$-lines) related to anesthesia in the majority of patients. Interstitial syndrome was detected in six patients in different study time frames.

LUS assessment has previously been described in several critical care studies. In these studies, researchers used ultrasound to compare lung isolation techniques, assist with endotracheal intubation or during cricothyroidotomy. However, there is lack of data describing LUS patterns and their perioperative changes during anesthesia [13].

Ventilator induced lung injury include biotrauma, atelectrauma, barotrauma and volutrauma [21]. Lung injury is associated with mechanical power and relates to tidal volume, driving pressure, respiratory rate and positive end expiratory pressure [22].

Our study indicates that even short time intraoperative lung ventilation in group of patients with low risk of PPC [23] may induce development of abnormal LUS findings.

Results of this study show dynamic changes in LUS patterns when the LUS is performed at different intervals during general anesthesia. Several factors may contribute to B-lines during anesthesia including gravity, blood accumulation and increased lung water [18]. The increase in the appearance of B-lines and in the number of positive regions may be related to lung de-aeration during anesthesia $[16,18]$. In our study we observed B-lines detected more often during anesthesia in upper areas of 8 zone protocol when patients were positioned in Trendelenburg position. This may suggest different cephalocaudal distribution of ventilation during anesthesia in Trendelenburg position. 
Not all changes resolved within two hours after recovery from anesthesia; however, none of the patients presented any sings of short term PPC. The interstitial syndrome criteria [19] were met in six patients at different time frames, however the subgroup of patients was very heterogeneous. Abnormal LUS findings may affect interpretation of the LUS results in cases when patients develop PPC.

Lung consolidation found in LUS may be related to atelectasis developing through either compression of lung parenchyma or resorption of alveolar gas. Their localization would be mainly in dependent lung areas not accessible in 8 zone protocol [24], however in our study we were able to detect them which may result either from their major extent in perioperative period or their specific location in pericardiac area. In a similar group of patients undergoing laparoscopic surgery in the Trendelenburg position absorption atelectasis occurred more frequently with higher $\mathrm{FiO}_{2}$ used during recruitment maneuvers [25]. Consolidations found before induction of anesthesia may result from hypoventilation secondary to premedication with benzodiazepines. Signs of atelectasis and pleural effusion in ASA 1-2 patients were identified intraoperatively by Chun et al. when assessed with electrical impedance lung tomography [26]. The LUS pattern changes may be related to low PEEP levels observed in the study. However, optimal level of PEEP during intraoperative ventilation remains unknown [26-28].

This is a pilot study and has several limitations. First, was the inability to perform LUS assessment of posterior regions of the lungs, due to the ongoing surgical procedure. Further evaluation of the lung-dependent regions may reveal additional pattern changes to those revealed using only the 8-zone protocol. Secondly, LUS assessments were only conducted over short time intervals, which resulted from duration of surgery, instead of monitoring the patients for longer times during and post anesthesia. The number of pulmonary complications as well as additional LUS changes may increase in group of patients with risk factors, the study population was composed of patients without respiratory abnormalities [23]. Our study due to sample size was not designed to identify factors contributing to the LUS pattern changes. Thirdly, this study did not test patients' hemodynamic status, a status that may also play a role in LUS changes [29, 30]. Finally, different LUS settings and equipment than that used in this study may reveal other pattern differences [31].

\section{CONCLUSIONS}

In conclusion, abnormal LUS findings may occur during uneventful anesthesia in a healthy cohort of patients. This may significantly influence the interpretation of LUS results in cases of perioperative pulmonary complications.

\section{Conflicts of interest}

The authors declare no conflict of interest.

\section{REFERENCES}

1. Ashton-Cleary DT. Is thoracic ultrasound a viable alternative to conventional imaging in the critical care setting? Br J Anaesth. 2013; 111(2): 152-160, doi: 10.1093/bja/aet076, indexed in Pubmed: 23585400.

2. Lichtenstein $D$. Ultrasound examination of the lungs in the intensive care unit. Pediatric Critical Care Medicine. 2009; 10(6): 693-698, doi: 10.1097/pcc.0b013e3181b7f637.

3. Al Deeb M, Barbic S, Featherstone R, et al. Point-of-care ultrasonography for the diagnosis of acute cardiogenic pulmonary edema in patients presenting with acute dyspnea: a systematic review and meta-analysis. Acad Emerg Med. 2014; 21(8): 843-852, doi: 10.1111/acem.12435, indexed in Pubmed: 25176151.

4. Buonsenso D, Moro $F$, Inchingolo $R$, et al. Effectiveness of rapid lung ultrasound training program for gynecologists and obstetricians managing pregnant women with suspected COVID-19. Ultrasound Obstet Gynecol. 2020; 56(1): 110-111, doi: 10.1002/uog.22066, indexed in Pubmed: 32349175.

5. Moro F, Buonsenso D, Moruzzi MC, et al. How to perform lung ultrasound in pregnant women with suspected COVID-19. Ultrasound Obstet Gynecol. 2020; 55(5): 593-598, doi: 10.1002/uog.22028, indexed in Pubmed: 32207208.

6. Buonsenso D, Raffaelli F, Tamburrini E, et al. Clinical role of lung ultrasound for diagnosis and monitoring of COVID-19 pneumonia in pregnant women. Ultrasound Obstet Gynecol. 2020; 56(1): 106-109, doi: 10.1002/uog.22055, indexed in Pubmed: 32337795.

7. Arbeid E, Demi A, Brogi E, et al. Lung Ultrasound Pattern Is Normal during the Last Gestational Weeks: An Observational Pilot Study. Gynecol Obstet Invest. 2017; 82(4): 398-403, doi: 10.1159/000448140, indexed in Pubmed: 27701165.

8. Inchingolo R, Smargiassi A, Moro F, et al. The diagnosis of pneumonia in a pregnant woman with coronavirus disease 2019 using maternal lung ultrasound. Am J Obstet Gynecol. 2020; 223(1): 9-11, doi: 10.1016/j. ajog.2020.04.020, indexed in Pubmed: 32360111.

9. Ambrozic J, Brzan Simenc G, Prokselj K, et al. Lung and cardiac ultrasound for hemodynamic monitoring of patients with severe pre-eclampsia. UItrasound Obstet Gynecol. 2017; 49(1): 104-109, doi: 10.1002/uog.17331, indexed in Pubmed: 27736042.

10. Krawczyk P, Jastrzębska A, Sałapa K, et al. Abnormal lung ultrasound pattern during labor: A prospective cohort pilot study. J Clin Ultrasound. 2019; 47(5): 261-266, doi: 10.1002/jcu.22692, indexed in Pubmed: 30729529.

11. Hemmes SNT, Gama de Abreu M, Pelosi P, et al. PROVE Network Investigators for the Clinical Trial Network of the European Society of Anaesthesiology. High versus low positive end-expiratory pressure during general anaesthesia for open abdominal surgery (PROVHILO trial): a multicentre randomised controlled trial. Lancet. 2014; 384(9942): 495-503, doi: 10.1016/S0140-6736(14)60416-5, indexed in Pubmed: 24894577.

12. Agostini P, Cieslik H, Rathinam S, et al. Postoperative pulmonary complications following thoracic surgery: are there any modifiable risk factors? Thorax. 2010; 65(9): 815-818, doi: 10.1136/thx.2009.123083, indexed in Pubmed: 20805178.

13. Bainbridge D, McConnell B, Royse C. A review of diagnostic accuracy and clinical impact from the focused use of perioperative ultrasound. Can J Anaesth. 2018; 65(4): 371-380, doi: 10.1007/s12630-018-1067-5, indexed in Pubmed: 29396742.

14. Nahler G. Lorentz-formula. Dictionary of Pharmaceutical Medicine. 2009: 107-107, doi: 10.1007/978-3-211-89836-9 803.

15. American Society of Anesthesiologists Physical Status Classification System. https://www.asahq.org/resources/clinical-information/asa-physical-status-classification-system (15 February 2019).

16. Bellani G, Rouby JJ, Constantin JM, et al. Looking closer at acute respiratory distress syndrome: the role of advanced imaging techniques. Curr Opin Crit Care. 2017; 23(1):30-37, doi: 10.1097/MCC.0000000000000380, indexed in Pubmed: 27906709.

17. Lichtenstein DA, Mezière GA. Relevance of lung ultrasound in the diagnosis of acute respiratory failure: the BLUE protocol. Chest. 2008; 134(1): 117-125, doi: 10.1378/chest.07-2800, indexed in Pubmed: 18403664.

18. Goffi A, Kruisselbrink R, Volpicelli $G$. The sound of air: point-of-care lung ultrasound in perioperative medicine. Can J Anaesth. 2018; 65(4): 399_ 416, doi: 10.1007/s12630-018-1062-x, indexed in Pubmed: 29411300. 
19. Volpicelli G, Elbarbary M, Blaivas $M$, et al. International Liaison Committee on Lung Ultrasound (ILC-LUS) for International Consensus Conference on Lung Ultrasound (ICC-LUS). International evidence-based recommendations for point-of-care lung ultrasound. Intensive Care Med. 2012; 38(4): 577-591, doi: 10.1007/s00134-012-2513-4, indexed in Pubmed: 22392031.

20. R Core Team. A language and environment for statistical computing. R Foundation for Statistical Computing, Vienna, Austria. https:// www.R-project.org/ (18 December 2018).

21. O'Gara B, Talmor D. Perioperative lung protective ventilation. BMJ. 2018; 362: k3030, doi: 10.1136/bmj.k3030, indexed in Pubmed: 30201797.

22. Gattinoni L, Tonetti T, Cressoni M, et al. Ventilator-related causes of lung injury: the mechanical power. Intensive Care Med. 2016; 42(10): 15671575, doi: 10.1007/s00134-016-4505-2, indexed in Pubmed: 27620287.

23. Neto AS, da Costa LG, Hemmes SNT, et al. LAS VEGAS. The LAS VEGAS risk score for prediction of postoperative pulmonary complications: An observational study. Eur J Anaesthesiol. 2018; 35(9): 691-701, doi: 10.1097/EJA.0000000000000845, indexed in Pubmed: 29916860.

24. Bellani G, Rouby JJ, Constantin JM, et al. Looking closer at acute respiratory distress syndrome: the role of advanced imaging techniques. Curr Opin Crit Care. 2017; 23(1):30-37, doi: 10.1097/MCC.0000000000000380, indexed in Pubmed: 27906709.

25. Kim BoR, Lee $\mathrm{S}$, Bae H, et al. Lung ultrasound score to determine the effect of fraction inspired oxygen during alveolar recruitment on absorption atelectasis in laparoscopic surgery: a randomized controlled trial. BMC
Anesthesiol. 2020; 20(1): 173, doi: 10.1186/s12871-020-01090-y, indexed in Pubmed: 32682397.

26. Chun $\mathrm{EH}$, Baik HJ, Moon HS, et al. Comparison of low and high positive end-expiratory pressure during low tidal volume ventilation in robotic gynaecological surgical patients using electrical impedance tomography: A randomised controlled trial. Eur J Anaesthesiol. 2019; 36(9): 641-648, doi: 10.1097/EJA.0000000000001047, indexed in Pubmed: 31306184.

27. Neto AS, Hemmes S, Barbas $C$, et al. Protective versus Conventional Ventilation for Surgery. Anesthesiology. 2015; 123(1): 66-78, doi: 10.1097/aln.0000000000000706.

28. Neto AS, Schultz M, Abreu MG. Intraoperative ventilation strategies to prevent postoperative pulmonary complications: Systematic review, meta-analysis, and trial sequential analysis. Best Practice \& Research Clinical Anaesthesiology. 2015; 29(3): 331-340, doi: 10.1016/j.bpa.2015.09.002.

29. Lichtenstein D. FALLS-protocol: lung ultrasound in hemodynamic assessment of shock. Heart Lung Vessel. 2013; 5(3): 142-147, indexed in Pubmed: 24364005.

30. Lichtenstein DA, Mezière GA, Lagoueyte JF, et al. A-lines and B-lines: lung ultrasound as a bedside tool for predicting pulmonary artery occlusion pressure in the critically ill. Chest. 2009; 136(4): 1014-1020, doi: 10.1378/chest.09-0001, indexed in Pubmed: 19809049.

31. Dietrich $C F$, Mathis $G$, Blaivas $M$, et al. Lung $B$-line artefacts and their use. J Thorac Dis. 2016; 8(6): 1356-1365, doi: 10.21037/jtd.2016.04.55, indexed in Pubmed: 27293860. 